\title{
Solvable lattice models labelled by Dynkin diagrams
}

\author{
S. Ole Warnaar*and Bernard Nienhuis ${ }^{\dagger}$ \\ Instituut voor Theoretische Fysica \\ Universiteit van Amsterdam \\ Valckenierstraat 65 \\ 1018 XE Amsterdam \\ The Netherlands
}

ITFA 93-01

\begin{abstract}
An equivalence between generalised restricted solid-on-solid (RSOS) models, associated with sets of graphs, and multi-colour loop models is established. As an application we consider solvable loop models and in this way obtain new solvable families of critical RSOS models. These families can all be classified by the Dynkin diagrams of the simply-laced Lie algebras. For one of the RSOS models, labelled by the Lie algebra pair $\left(\mathrm{A}_{L}, \mathrm{~A}_{L}\right)$ and related to the $\mathrm{C}_{2}^{(1)}$ vertex model, we give an off-critical extension, which breaks the $Z_{2}$ symmetry of the Dynkin diagrams.
\end{abstract}

*e-mail: warnaar@phys.uva.nl

${ }_{\dagger}^{\dagger}$ e-mail: nienhuis@phys.uva.nl 


\section{Introduction}

In recent years many solutions to the star-triangle or Yang-Baxter equation (YBE) [1] have been found. Among these solutions, the A-D-E lattice models, found by Pasquier [2], have drawn particular attention. Pasquier showed, in fact, that to any arbitrary graph one can associate a solvable restricted solid-on-solid (RSOS) model. Requiring criticality led to the restriction to graphs which are Dynkin diagrams of the simply-laced A-D-E Lie algebras. An important feature of the A-D-E models is that they can all be mapped onto the same polygon or loop model, which in turn is equivalent to the 6 -vertex model.

Recently, by extending Pasquier's and similar methods of Owczareck and Baxter [3], Warnaar et al. [4 found a new family of models associated with graphs. Via a different approach these same models were also found by Roche [5], who suggested the name dilute A-D-E models. Again, the whole family of dilute A-D-E models can be mapped onto a single loop model, the $\mathrm{O}(n)$ model [6], which is related to the 19-vertex vertex model of Izergin and Korepin [7].

In this paper we further exploit the relation between RSOS models related to graphs and loop models. We define a general multi-colour loop (MCL) model and show its equivalence with a RSOS model defined by arbitrary sets of graphs. Then we consider several examples for which these models are actually solvable, and find, besides the known A-D-E and dilute A-D-E models, new families of critical RSOS models labelled by Dynkin diagrams. As a further generalisation we also consider models of mixed loop-vertex type. Finally, for one of the examples, related to the

$\mathrm{C}_{2}^{(2)}$ Lie algebra, we present an off-critical extension. This extension has the property that it breaks the $\mathrm{Z}_{2}$ symmetry of the underlying Dynkin diagrams. In the Appendix we describe the YBE for loop models [8] and show how it relates to the YBE for the RSOS model.

\section{Multi-colour loop model}

We consider a square lattice $\mathcal{L}$. Each edge of $\mathcal{L}$ can either be occupied by a line segment, that has one of $C$ possible colours, or be empty. Line segments of equal colour on adjoining edges must form closed polygons or loops. A configuration $G$ is defined as a collection of coloured loops on $\mathcal{L}$, with the restriction that polygons of the same colour do not intersect. An example of a configuration is given in figure 2 . The total number of allowed vertices $V$ is given by $V=3 C^{2}+5 C+1$. For $C=1$ and $C=2$ all possible vertices are shown in figure 1(a) and (b) respectively. A loop of colour $i$ has fugacity $n_{i}$ and the Boltzmann weight of vertex $k$ is given by $\rho_{k}$. The partition function of the MCL model is defined as

$$
Z=\sum_{G} \rho_{1}^{m_{1}} \ldots \rho_{V}^{m_{V}} n_{1}^{p_{1}} \ldots n_{C}^{p_{C}}
$$

where $p_{i}$ is the total number of loops of colour $i$ and $m_{k}$ the number of vertices of type $k$. For $C=1$ the MCL model coincides with the loop model defined in [⿴囗十).

If the weights $\rho_{1}, \ldots, \rho_{6 C+1}$ are all zero, only configurations that densely cover the entire lattice give a non-zero contribution to the partition function. Such loop 
models we call dense, opposed to so-called dilute loop models which allow for edges of $\mathcal{L}$ to be unoccupied.

(a)

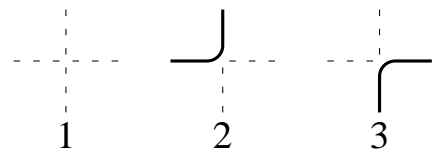

(b)
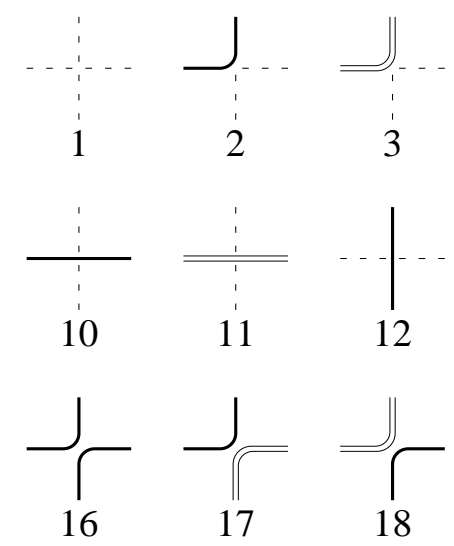
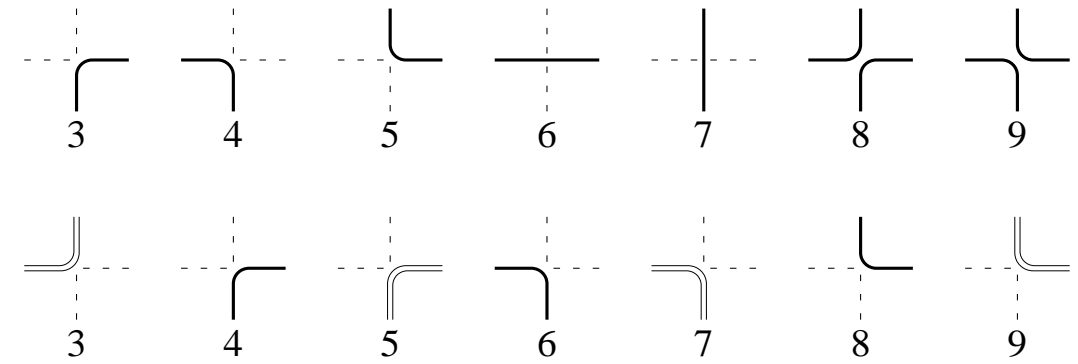

9
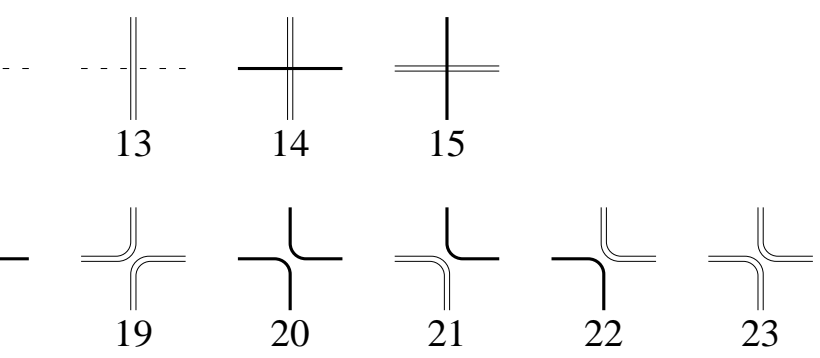

Figure 1: (a) The 9 vertices of the $C=1$ MCL model and (b) the 23 vertices for the $C=2$ case.

\section{$3 \quad$ RSOS model}

In this section we define a restricted solid-on-solid model and show that its partition function equals that of the multi-colour loop model.

\subsection{Definition of the model}

Consider an arbitrary connected graph $\mathcal{G}_{i}$. Such a graph consists of a set of $L_{i}$ nodes, labelled by an integer height $a_{i} \in\left\{1, \ldots, L_{i}\right\}$ and a number of bonds between the nodes. We do not allow more than one bond between two nodes. Two nodes are called adjacent $(\sim)$ on $\mathcal{G}_{i}$ if they are connected via a single a bond. A graph is called simple if it has no nodes connected to themselves. Examples of simple and non-simple connected graphs are shown in figure 1 and 5 respectively.

We can represent the graph $\mathcal{G}_{i}$ by an adjacency matrix $A^{i}$ as follows

$$
A_{a_{i}, b_{i}}^{i}= \begin{cases}1 & a_{i} \sim b_{i} \\ 0 & \text { otherwise. }\end{cases}
$$

We denote the largest eigenvalue of $A^{i}$ by $\Lambda_{i}$ and the corresponding eigenvector by $S^{i}$.

We now take $C$ such arbitrary graphs, labelled $\mathcal{G}_{1}, \ldots, \mathcal{G}_{C}$. Let $a$ be the $C$-dimensional vector $a=\left(a_{1}, \ldots, a_{C}\right)$ and define

$$
S_{a}=\prod_{i} S_{a_{i}}^{i}
$$




$$
A_{a, b}^{i}=A_{a_{i}, b_{i}}^{i} \prod_{j \neq i} \delta_{a_{j}, b_{j}}
$$

where we use the convention that sums and products over $i$ and $j$ always range from 1 to $C$. With the above definitions the Boltzmann weight of an elementary face of the RSOS model is defined as

$$
\begin{aligned}
W\left(\begin{array}{ll}
d & c \\
a & b
\end{array}\right) & =\rho_{1} \delta_{a, b, c, d}+\delta_{a, b, c} \sum_{i} \rho_{i+1} A_{a, d}^{i} \\
& +\delta_{a, c, d} \sum_{i} \rho_{i+C+1} A_{a, b}^{i}+\left(\frac{S_{a}}{S_{b}}\right)^{1 / 2} \delta_{b, c, d} \sum_{i} \rho_{i+2 C+1} A_{a, b}^{i} \\
& +\left(\frac{S_{c}}{S_{a}}\right)^{1 / 2} \delta_{a, b, d} \sum_{i} \rho_{i+3 C+1} A_{a, c}^{i}+\delta_{a, b} \delta_{c, d} \sum_{i} \rho_{i+4 C+1} A_{a, d}^{i} \\
& +\delta_{a, d} \delta_{b, c} \sum_{i} \rho_{i+5 C+1} A_{a, b}^{i}+\sum_{i \neq j} \rho_{k(i, j)} A_{a, d}^{i} A_{a, b}^{j} \\
& +\delta_{a, c} \sum_{i, j} \rho_{l(i, j)} A_{a, b}^{i} A_{a, d}^{j}+\left(\frac{S_{a} S_{c}}{S_{b} S_{d}}\right)^{1 / 2} \delta_{b, d} \sum_{i, j} \rho_{m(i, j)} A_{a, b}^{i} A_{b, c}^{j},
\end{aligned}
$$

where the $i$-th component of the height vectors $a, b, c$ and $d$ can take any of the $L_{i}$ heights on $\mathcal{G}_{i}$. The generalised Kronecker $\delta$ and the functions $k, l$ and $m$ used above are given by

$$
\begin{aligned}
\delta_{p, q, \ldots, s} & =\prod_{i} \delta_{p_{i}, q_{i}} \ldots \delta_{p_{i}, s_{i}} \\
k(i, j) & =(C-1) i+j-\theta(j-i)+5 C+2 \\
l(i, j) & =C i+j+C^{2}+4 C+1 \\
m(i, j) & =C i+j+2 C^{2}+4 C+1,
\end{aligned}
$$

with $\theta$ the step function:

$$
\theta(x)= \begin{cases}0 & x<0 \\ 1 & x \geq 1\end{cases}
$$

We note that the total number of terms in equation (3.3) is $V$.

In analogy with the MCL model, if $\rho_{1}, \ldots, \rho_{6 C+1}$ are all zero, we name the RSOS model dense. For such models, if all adjacency graphs are simple, neighbouring sites of the lattice must have different height. So-called dilute RSOS models allow for neighbouring sites of $\mathcal{L}$ to have equal height.

As will become clear in the following, we require for dilute RSOS models that all $C$ adjacency graphs are simple.

\subsection{MCL-RSOS equivalence}

We now show that the partition function of the RSOS model, given by

$$
Z=\sum_{\text {heights faces }} \prod_{W} W\left(\begin{array}{ll}
d & c \\
a & b
\end{array}\right),
$$


where the product is over all faces of the square lattice $\mathcal{L}$, can be mapped onto that of the MCL model. The method is a straightforward generalisation of the work of Pasquier [2], Owczarek and Baxter [3] and Warnaar et al. [四].

As a first step we substitute the expression for the Boltzmann weight (3.3) and expand the above partition function into a sum over $V^{N}$ terms, where $N$ is the number of faces of the lattice. For each face of $\mathcal{L}$, a given term in the expansion has one of the $V$ terms of equation (3.3). These $V$ possible terms can be represented diagrammatically as shown in figure 1(a) and (b) for the $C=1$ and $C=2$ case respectively. A line of colour $i$, separating two neighbouring sites with heights $a$ and $b$ respectively, implies

$$
\begin{aligned}
& a_{i} \sim b_{i} \\
& a_{j}=b_{j} \quad j \neq i .
\end{aligned}
$$

We now have to distinguish between dense and dilute RSOS models.

For dilute RSOS models, since we do not allow for non-simple graphs, all diagonal elements of the $C$ adjacency matrices are zero. Consequently, $a_{i} \sim b_{i}$ means that $a_{i} \neq b_{i}$, and hence that a line separating two neighbouring sites can be viewed as a domain wall separating two neighbouring sites with different heights. As a results of this and the $\delta$-functions in (3.3), only configurations in which lines of the same colour join together, to form domain walls separating regions of the lattice with different height, give a non-zero contribution to the partition function. A typical configuration is shown in figure 2. If we had allowed for non-simple graphs, configurations where domain walls would simply end somewhere on the lattice would not give a vanishing contribution.

For dense RSOS models we do not have this complication. All edges of the lattice are occupied by polygon segments and domain walls therefore cannot end. Besides simple graphs, we can now allow for non-simple adjacency graphs as well. If adjacency graph $\mathcal{G}_{i}$ is non-simple, a line of colour $i$ separating two neighbouring sites does not necessarily separate two sites with different height. For simplicity we will still refer to such a line as a (local) domain wall. Due to the $\delta$-functions we again have that only configurations in which lines of the same colour join together, to form global domain walls, give a non-zero contribution to the partition function.

The partition function is now given as the sum over all configurations $G$ of domain walls and a sum over heights consistent with $G$

$$
Z=\sum_{G} \rho_{1}^{m_{1}} \ldots \rho_{V}^{m_{V}} \sum_{\text {heights }} \prod_{i} \prod_{a_{i}, b_{i}=1}^{L_{i}}\left(\frac{S_{b_{i}}^{i}}{S_{a_{i}}^{i}}\right)^{m_{b_{i} a_{i}}}
$$

where we have used the factorisation property (3.2) of $S_{a}$ and the meaning of a domain wall of colour $i$, as formulated in (3.7). The integer $m_{b_{i} a_{i}}$ denotes the total power of $S_{b_{i}} / S_{a_{i}}$ arising from the vertices of type $\rho_{2 C+2}, \ldots, \rho_{4 C+1}$ and $\rho_{2 C^{2}+5 C+2}, \ldots, \rho_{V}$, where we count the powers of $S_{b_{i}} / S_{a_{i}}$ and $S_{a_{i}} / S_{b_{i}}$ separately.

To avoid technical difficulties, we assume that all boundary sites of $\mathcal{L}$ carry the same height vector. (For the treatment of other boundary conditions see [2, 8].) All domain walls then form closed polygons or loops. Polygons may of course surround 
other polygons of any colour, but can only be intersected by polygons of a different colour. If a polygon of colour $i$ is intersected by other polygons, the height vectors immediately inside and outside this polygon are not unique. However, the $i$-th component of these vectors does have a unique value. We call these the inner and outer height of the polygon respectively. We now make the following decomposition:

$$
\begin{gathered}
i \frac{a \| c}{b\rceil a} j=i \frac{a}{b\rceil a} \times \frac{a \| \frac{c}{a} j}{\left(\frac{S_{b_{i}}^{i} S_{c_{j}}^{j}}{S_{a_{i}}^{i} S_{a_{j}}^{j}}\right)^{1 / 2}}\left(\frac{S_{b_{i}}^{i}}{S_{a_{i}}^{i}}\right)^{1 / 2}\left(\frac{S_{c_{j}}^{j}}{S_{a_{j}}^{j}}\right)^{1 / 2},
\end{gathered}
$$

where the labels $i$ and $j$ in the diagrams denote colours. As a result, the total contribution to $m_{a_{i} b_{i}}-m_{b_{i} a_{i}}$ of a polygon of colour $i$, with inner height $a_{i}$ and outer height $b_{i}$ is always 1 .

We can now perform the summation over the height vectors in (3.8) for each component independently. When summing over the $i$ height components, we start with polygons of colour $i$ which do not surround other polygons of the same colour. If such a polygon has inner height $a_{i}$ and outer height $b_{i}$, we get

$$
\sum_{a_{i} \sim b_{i}} \frac{S_{a_{i}}^{i}}{S_{b_{i}}^{i}}=\sum_{a_{i}=1}^{L_{i}} A_{b_{i}, a_{i}}^{i} \frac{S_{a_{i}}^{i}}{S_{b_{i}}^{i}}=\Lambda_{i} .
$$

The result is that these polygons contribute a factor $\Lambda_{i}$ and that their dependence on the outer height $b_{i}$ disappears. Therefore, the summation over the $i$-th height component of the regions immediately outside these polygons can now be performed in the same way. Repeating this process from inside out and we obtain, after completely summing out the $i$-th height component,

$$
\Lambda_{i}^{p_{i}}
$$

where $p_{i}$ is the number of polygons of colour $i$.

If we perform the summation for all $C$ height components, and make the identification $\Lambda_{i}=n_{i}$ we find that the partition function of the RSOS model is that of the MCL model.

The equivalence between the partition functions of the MCL and RSOS model holds irrespective of the solvability of the models. Clearly, as a consequence of the equivalence, if either one of the models is solvable, in the sense that we can compute its partition function, the other model is solvable as well. In the Appendix we show that if the MCL model satisfies the YBE, then, as an immediate consequence, the YBE for the RSOS model holds as well.

\section{Solvable Examples}

We now consider several special cases for which the MCL model and hence the RSOS model is solvable. By a solvable MCL model we mean that it satisfies the YBE for loop models [8], which is described in some detail in Appendix A.1. 


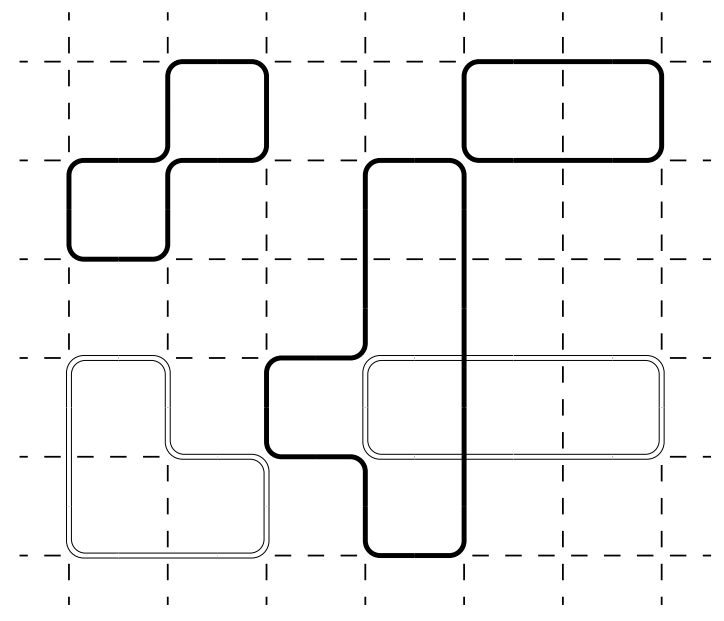

Figure 2: A polygon configuration. Only loops of different colour may intersect

Two of these cases are already known in the literature and are only presented for completeness. All examples are either 1-, or 2-colour loop models. So far we have not been able to find any non-trivial solvable MCL model with more than two colours.

In Appendix A we give an alternative equivalence between the MCL model and the RSOS model on the level of the YBE.

The Temperley-Lieb (TL) loop model This dense loop model, which first occurred in the mapping of the $q$-state self-dual Potts model onto the 6 -vertex model [9], is given by equation (2.1) with $C=1$. The weights $\rho_{1}, \ldots, \rho_{9}$ of the vertices, shown in figure 1(a), and the fugacity $n_{1}=\sqrt{q}$ are given by

$$
\rho_{1}=\ldots=\rho_{7}=0 \quad \rho_{8}=\frac{\sin (\lambda-u)}{\sin \lambda} \quad \rho_{9}=\frac{\sin u}{\sin \lambda} \quad n_{1}=2 \cos \lambda .
$$

It is this model for which the equivalence between the RSOS and loop model was first established [2, 3].

The $\mathbf{O}(\boldsymbol{n})$ model [6] This is a dilute loop model related to the Izergin-Korepin or $\mathrm{A}_{2}^{(2)}$ vertex model [7]. It is the most general 1-colour loop model of the form (2.1). Again, the dilute RSOS models based on this model have been constructed before in

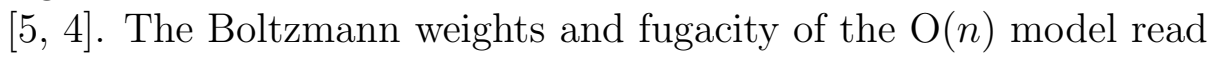

$$
\begin{aligned}
& \rho_{1}=\frac{\sin 2 \lambda \sin 3 \lambda+\sin u \sin (3 \lambda-u)}{\sin 2 \lambda \sin 3 \lambda} \quad \rho_{2}=\rho_{3}=\frac{\sin (3 \lambda-u)}{\sin 3 \lambda} \\
& \rho_{4}=\rho_{5}=\epsilon_{1} \frac{\sin u}{\sin 3 \lambda} \quad \rho_{6}=\rho_{7}=\epsilon_{2} \frac{\sin u \sin (3 \lambda-u)}{\sin 2 \lambda \sin 3 \lambda} \\
& \rho_{8}=\frac{\sin (2 \lambda-u) \sin (3 \lambda-u)}{\sin 2 \lambda \sin 3 \lambda} \quad \rho_{9}=-\frac{\sin u \sin (\lambda-u)}{\sin 2 \lambda \sin 3 \lambda} \quad n_{1}=-2 \cos 4 \lambda,
\end{aligned}
$$

where, here and in the following, $\epsilon_{1}^{2}=\epsilon_{2}^{2}=1$. 
The $\mathbf{C}_{2}^{(1)}$ loop model Whereas the $\mathrm{O}(n)$ model is the natural dilute generalisation of the TL model model, so the $\mathrm{C}_{2}^{(1)}$ model can be seen as the simplest non-trivial generalisation of the TL model to a model with more than one colour. It is a dense 2-colour loop model with vertices shown in figure 1(b) and weights

$$
\begin{aligned}
& \rho_{1}=\ldots=\rho_{13}=0 \quad \rho_{14}=\rho_{15}=\epsilon_{1} \frac{\sin u \sin (3 \lambda-u)}{\sin \lambda \sin 3 \lambda} \\
& \rho_{16}=\rho_{19}=\frac{\sin (\lambda-u) \sin (3 \lambda-u)}{\sin \lambda \sin 3 \lambda} \quad \rho_{17}=\rho_{18}=\frac{\sin (3 \lambda-u)}{\sin 3 \lambda} \\
& \rho_{20}=\rho_{23}=-\frac{\sin u \sin (2 \lambda-u)}{\sin \lambda \sin 3 \lambda} \quad \rho_{21}=\rho_{22}=\epsilon_{2} \frac{\sin u}{\sin 3 \lambda} \\
& n_{1}=n_{2}=-2 \cos 2 \lambda .
\end{aligned}
$$

By making an arrow covering of the polygons, as described in section E, this model maps onto the $\mathrm{C}_{2}^{(1)}$ vertex model of [10].

The $\mathbf{A}_{2}^{(1)}$ loop model This dilute model, related to the $A_{2}^{(1)}$ vertex model found in [11, 10, is given by (2.1) with $C=1$ and

$$
\begin{aligned}
& \rho_{1}=\rho_{8}=\frac{\sin (\lambda-u)}{\sin \lambda} \quad \rho_{2}=\rho_{3}=1 \quad \rho_{4}=\rho_{5}=0 \\
& \rho_{6}=\rho_{7}=\epsilon_{1} \frac{\sin u}{\sin \lambda} \quad \rho_{9}=\frac{\sin u}{\sin \lambda} \quad n_{1}=2 \cos \lambda .
\end{aligned}
$$

The $\mathbf{A}_{3}^{(1)}$ loop model This dense 2-colour loop model is related to the $\mathrm{A}_{3}^{(1)}$ vertex model of [11, 10]. The weights are given by

$$
\begin{aligned}
& \rho_{1}=\ldots=\rho_{13}=\rho_{21}=\rho_{22}=0 \quad \rho_{14}=\rho_{15}=\epsilon_{1} \frac{\sin u}{\sin \lambda} \\
& \rho_{16}=\rho_{19}=\frac{\sin (\lambda-u)}{\sin \lambda} \quad \rho_{17}=\rho_{18}=1 \quad \rho_{20}=\rho_{23}=\frac{\sin u}{\sin \lambda} \\
& n_{1}=n_{2}=2 \cos \lambda .
\end{aligned}
$$

We remark that, though the $A_{1}^{(1)}-A_{3}^{(1)}$ vertex models all relate to loop models, this does not seem to be true for the $\mathrm{A}_{n}^{(1)}$ family [11, 10] in general.

\section{$5 \quad$ Mixed models}

Provided that polygon segments of the same colour do not intersect, each loop model can be mapped onto a vertex model [9]. We cover loops of colour $i$ by arrows of that same colour, such that the loops become oriented. Following a loop in the direction 
of the arrows, we assign a phase factor $s_{i}$ to a turn to the left and a factor $s_{i}^{-1}$ to a turn to the right, where $s_{i}$ is defined by $n_{i}=s_{i}^{4}+s_{i}^{-4}$. Summing over all possible arrow coverings of a configuration, each polygon of colour $i$ acquires a total factor $n_{i}$. Interchanging the summation over all loop configurations and over all arrow coverings, the sum over the loops can readily be performed. The resulting partition function is that of a vertex model, where the arrows arround a vertex obey the ice-rule for each colour independently.

In general, the inverse of the above mapping is not possible. Only very few solvable vertex models, that satisfy an ice-rule, admit a loop interpretation. Nevertheless, many vertex models allow for a partial mapping onto a loop model. That is, some vertex degrees of freedom can be converted into loop degrees of freedom, but not all. Via the MCL-RSOS correspondence, these mixed loop-vertex models can be mapped onto $R S O S$-vertex or equivalently $R S O S$-SOS models.

We shall not try to give a complete description off all models that allow such a procedure, but consider as an example the $\mathrm{A}_{3}^{(2)}$ vertex model. For the definition of this 36-vertex model we refer to [10]. The equivalent loop-vertex model has 20 vertices, shown in figure 3, with weights and fugacity

$$
\begin{aligned}
& \rho_{1}=\ldots=\rho_{4}=\epsilon_{1} \frac{\sin u \cos (2 \lambda-u)}{\sin \lambda \cos 2 \lambda} \quad \rho_{5}=\rho_{6}=\rho_{7}=\frac{\sin (\lambda-u) \cos (2 \lambda-u)}{\sin \lambda \cos 2 \lambda} \\
& \rho_{8}=\ldots=\rho_{11}=\frac{\cos (2 \lambda-u)}{\cos 2 \lambda} \quad \rho_{12}=\rho_{13}=\rho_{14}=-\frac{\sin u \cos (\lambda-u)}{\sin \lambda \cos 2 \lambda} \quad(5.1) \\
& \rho_{15}=\ldots=\rho_{18}=\epsilon_{2} \frac{\sin u}{\cos 2 \lambda} \quad \rho_{19}=\rho_{20}=1 \quad n_{1}=-2 \cos 2 \lambda .
\end{aligned}
$$

Figure 3: The 20 vertices of the $\mathrm{A}_{3}^{(2)}$ loop-vertex model.

Via the MCL-RSOS equivalence, this model maps onto a RSOS-vertex model, where an elementary face of the lattice is denoted as

$$
W\left(\begin{array}{lll}
d & \gamma & c \\
\delta & & \beta \\
a & \alpha & b
\end{array}\right)=W\left(\begin{array}{ccc}
d_{1} & \gamma & c_{1} \\
\delta & & \beta \\
a_{1} & \alpha & b_{1}
\end{array}\right)=\ldots
$$

The latin indices label 1-dimensional height variables and the greek indices the arrows. We note that two neighbouring sites either have the same height separated by an arrow or have different heights.

To cast this into a somewhat nicer form, we make use of the SOS-vertex equivalence for ice-type models [12] to write this as a RSOS-SOS model. For that purpose 
we assign 2-dimensional height vectors $a$ to each site of the lattice. The first component of such a vector is one of the heights $a_{1} \in\left\{1, \ldots, L_{1}\right\}$. The second component is a height variable $a_{2} \in \mathbb{Z}$. Two neighbouring sites of the RSOS-vertex model, with heights $a_{1}$ and $b_{1}$ separated by an arrow, correspond to height vectors $a$ and $b$ of the RSOS-SOS model, with

$$
\begin{aligned}
& a_{1}=b_{1} \\
& a_{2}-b_{2}= \pm 1,
\end{aligned}
$$

and the convention that the height to the left of the arrow is highest. For two sites of the RSOS-vertex model that are not separated by an arrow, we get heights $a$ and $b$, with

$$
\begin{aligned}
& a_{1} \sim b_{1} \\
& a_{2}=b_{2} .
\end{aligned}
$$

If we define $A_{a, b}^{i}(i=1,2)$ and $S_{a}$ as in equation (3.2), where

$$
\begin{aligned}
& A_{a_{2}, b_{2}}^{2}=\delta_{a_{2}, b_{2}-1}+\delta_{a_{2}, b_{2}+1} \\
& S_{a_{2}}^{2}=1 \\
& a_{2}, b_{2} \in \mathbb{Z}
\end{aligned}
$$

we finally get for the $\mathrm{A}_{3}^{(2)}$ SOS model

$$
\begin{aligned}
W\left(\begin{array}{ll}
d & c \\
a & b
\end{array}\right) & =\rho_{1} \sum_{i \neq j} A_{a, b}^{i} A_{a, d}^{j}+\delta_{a, c}\left(\rho_{5} \sum_{i=j}+\rho_{8} \sum_{i \neq j}\right) A_{a, b}^{i} A_{a, d}^{j} \\
& +\delta_{b, d}\left(\frac{S_{a} S_{c}}{S_{b} S_{d}}\right)^{1 / 2}\left(\rho_{12} \sum_{i=j}+\rho_{15} \sum_{i \neq j}\right) A_{a, b}^{i} A_{b, c}^{j} \\
& +\left(\rho_{19}-\rho_{5}-\rho_{12}\right) \delta_{a, c} \delta_{b, d} A_{a, b}^{2} A_{a, d}^{2} .
\end{aligned}
$$

Similarly, we can construct mixed SOS models starting from other vertex models. For the solutions of the YBE found in 10 we get models with height variables that have $r$ restricted and $u$ unrestricted components, with $r$ and $u$ listed below

$$
\begin{array}{llll}
\mathrm{A}_{2 n-1}^{(1)}: & r=2 & u=n-2 & (n \geq 2) \\
\mathrm{A}_{2 n}^{(1)}: & r=1 & u=n-1 & \\
\mathrm{C}_{n}^{(1)}: & r=2 & u=n-2 & (n \geq 2) \\
\mathrm{A}_{2 n-1}^{(2)}: & r=1 & u=n-1 & \\
\mathrm{~A}_{2 n}^{(2)}: & r=1 & u=n-1 . &
\end{array}
$$

\section{A-D-E classification}

All loop models presented in the previous sections are critical when $n_{i} \leq 2$. For $n_{i}>2$ the trigonometric functions have to be replaced by hyperbolic functions and the models become non-critical and in some cases even complex. It is therefore 
natural to restrict the graphs $\mathcal{G}_{i}$ of the RSOS models, to those that have adjacency matrices $A^{i}$ with largest eigenvalue $\Lambda_{i}$ less or equal than two. In fact, all simple connected graphs with $\Lambda_{i} \leq 2$ have been classified [13] and are given by the Dynkin diagrams of the classical and affine simply-laced Lie algebras, shown in figure 1 . For

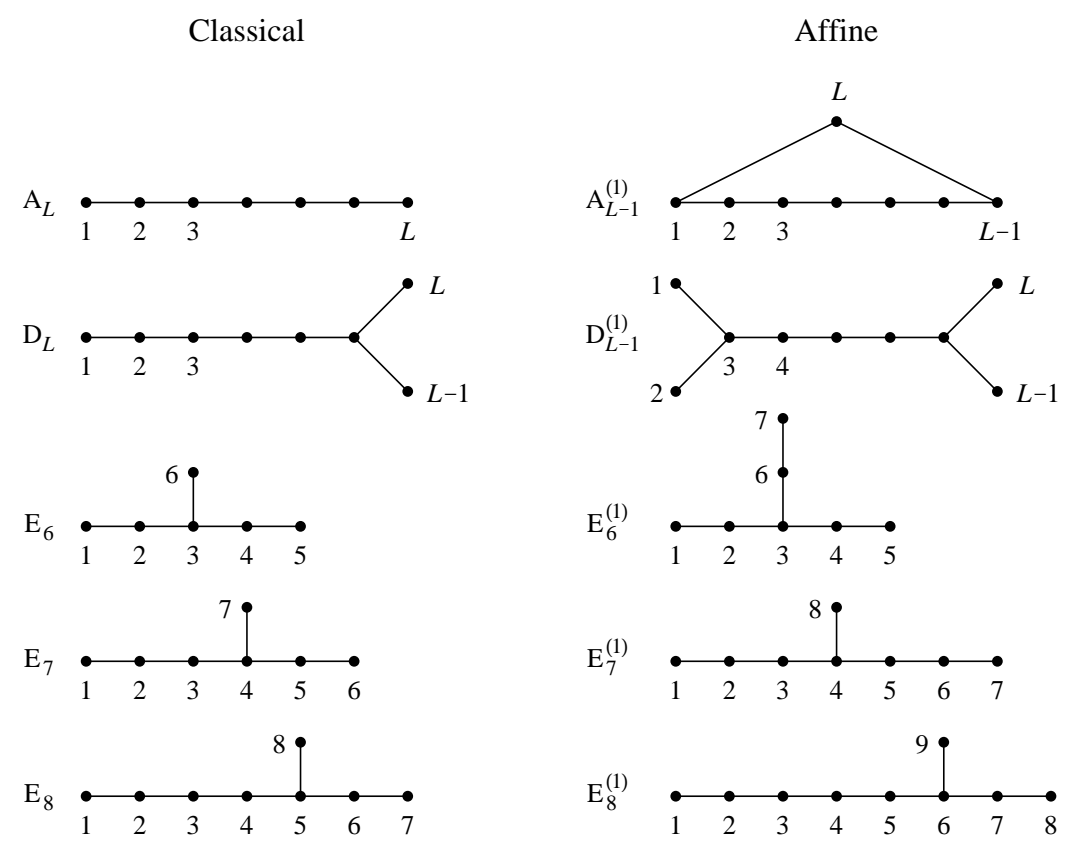

Figure 4: Dynkin diagrams of the simply-laced Lie algebras.

the classical algebras, the largest eigenvalue of the adjacency matrix of its Dynkin diagram is given by $2 \cos \frac{\pi}{h}$, where $h$ is the Coxeter number of the algebra. For the affine algebras the largest eigenvalue is 2 . The respective values of $h$, and the largest eigenvectors are given in table 1 for each algebra. In the case of RSOS models, we also alow for non-simple connected graphs. However, none of the graphs with largest eigenvalue $\leq 2$, leads to intrinsically new models, as they can always be viewed as one of the simple graphs where a $\mathrm{Z}_{2}$ symmetry is modded out, see figure 5 .
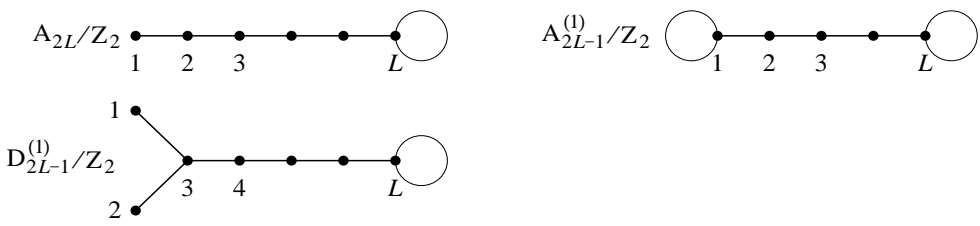

Figure 5: Non-simple connected graphs with largest eigenvalue $\leq 2$.

\section{Off-critical models}

It is well-known that all critical A-D models based on the Temperley-Lieb loop model admit an extension away from criticality while remaining solvable. The off-critical 


\begin{tabular}{|c|c|c|}
\hline algebra & $h$ & Perron-Frobenius vector \\
\hline $\mathrm{A}_{L}$ & $L+1$ & $\left(\sin \frac{\pi}{h}, \sin \frac{2 \pi}{h}, \ldots, \sin \frac{L \pi}{h}\right)$ \\
\hline $\mathrm{D}_{L}$ & $2 L-2$ & $\left(2 \sin \frac{\pi}{h}, \ldots, 2 \sin \frac{(L-2) \pi}{h}, 1,1\right)$ \\
\hline $\mathrm{E}_{6}$ & 12 & $\left(\sin \frac{\pi}{h}, \ldots, \sin \frac{3 \pi}{h}, \frac{\sin \frac{5 \pi}{h}}{2 \cos \frac{\pi}{h}}, 2 \cos \frac{4 \pi}{h} \sin \frac{\pi}{h}, \frac{\sin \frac{3 \pi}{h}}{2 \cos \frac{\pi}{h}}\right)$ \\
\hline $\mathrm{E}_{7}$ & 18 & $\left(\sin \frac{\pi}{h}, \ldots, \sin \frac{4 \pi}{h}, \frac{\sin \frac{6 \pi}{h}}{2 \cos \frac{\pi}{h}}, 2 \cos \frac{5 \pi}{h} \sin \frac{\pi}{h}, \frac{\sin \frac{4 \pi}{h}}{2 \cos \frac{\pi}{h}}\right)$ \\
\hline $\mathrm{E}_{8}$ & 30 & $\left(\sin \frac{\pi}{h}, \ldots, \sin \frac{4 \pi}{h}, \frac{\sin \frac{6 \pi}{h}}{2 \cos \frac{\pi}{h}}, 2 \cos \frac{6 \pi}{h} \sin \frac{\pi}{h}, \frac{\sin \frac{5 \pi}{h}}{2 \cos \frac{\pi}{h}}\right)$ \\
\hline $\mathrm{A}_{L-1}^{(1)}$ & $L$ & $(1,1, \ldots, 1)$ \\
\hline $\mathrm{D}_{L-1}^{(1)}$ & $2 L-6$ & $(1,1,2, \ldots, 2,1,1)$ \\
\hline $\mathrm{E}_{6}^{(1)}$ & 6 & $(1,2,3,2,1,2,1)$ \\
\hline $\mathrm{E}_{7}^{(1)}$ & 12 & $(1,2,3,4,3,2,1,2)$ \\
\hline $\mathrm{E}_{8}^{(1)}$ & 30 & $(1,2,3,4,5,6,4,2,3)$ \\
\hline
\end{tabular}

Table 1: Coxeter number and largest eigenvector of the simply-laced Lie algebras

models based on the classical Lie algebra $\mathrm{A}_{L}$ for example, are the models of Andrews, Baxter and Forrester [14.

Recently, the off-critical extension of the dilute $\mathrm{A}_{L}$ models based on the $\mathrm{O}(n)$ model was found [4]. As an interesting feature, these models break a $\mathrm{Z}_{2}$ symmetry of the underlying Dynkin diagrams, when $L$ is odd.

A natural question therefore is: which of the new A-D-E models presented in section 4, admit an extension away from criticality? So far, we have not studied this problem in any systematic way. However, for the $\mathrm{C}_{2}^{(1)}$ RSOS model based on the Lie algebra pair $\left(\mathrm{A}_{L}, \mathrm{~A}_{L}\right)$, the extension can easily be found by making the transformation $a_{1} \lambda \rightarrow a_{1} \lambda+\frac{1}{2} i \ln p$ to the $\mathrm{C}_{2}^{(1)}$ RSOS model of Jimbo et al. 15.

This solutions involves the $\vartheta$-functions [16

$$
\begin{aligned}
& \vartheta_{1}(u)=2 p^{1 / 4} \sin u \prod_{n=1}^{\infty}\left(1-2 p^{2 n} \cos 2 u+p^{4 n}\right)\left(1-p^{2 n}\right) \\
& \vartheta_{4}(u)=\prod_{n=1}^{\infty}\left(1-2 p^{2 n-1} \cos 2 u+p^{4 n-2}\right)\left(1-p^{2 n}\right),
\end{aligned}
$$

where we have suppressed the dependence on the nome $p,|p|<1$. If we also define the unit-vectors $e_{1}=(1,0)$ and $e_{2}=(0,1)$, and use the notation $e_{-\mu}=-e_{\mu}, a_{-\mu}=-a_{\mu}$, $\mu= \pm 1, \pm 2$, where $a=\left(a_{1}, a_{2}\right)$, the solution reads

$$
\begin{aligned}
W\left(\begin{array}{cc}
a+e_{\mu} & a \\
a & a-e_{\mu}
\end{array}\right) & =\frac{\vartheta_{1}(\lambda-u) \vartheta_{1}(3 \lambda-u)}{\vartheta_{1}(\lambda) \vartheta_{1}(3 \lambda)} \\
W\left(\begin{array}{cc}
a & a-e_{\mu} \\
a+e_{\mu} & a
\end{array}\right) & =-\left(\frac{S\left(a+e_{\mu}\right) S\left(a-e_{\mu}\right)}{S^{2}(a)}\right)^{1 / 2} \frac{\vartheta_{1}(u) \vartheta_{1}(2 \lambda-u)}{\vartheta_{1}(\lambda) \vartheta_{1}(3 \lambda)}
\end{aligned}
$$




$$
\begin{aligned}
& W\left(\begin{array}{cc}
a+e_{\mu} & a \\
a & a-e_{\nu}
\end{array}\right)=\frac{\vartheta_{1}(3 \lambda-u) \vartheta_{4}\left(a_{\mu} \lambda-a_{\nu} \lambda+\lambda-u\right)}{\vartheta_{1}(3 \lambda) \vartheta_{4}\left(a_{\mu} \lambda-a_{\nu} \lambda+\lambda\right)} \\
& W\left(\begin{array}{cc}
a & a-e_{\nu} \\
a+e_{\mu} & a
\end{array}\right)=\epsilon_{2}\left(\frac{S\left(a+e_{\mu}\right) S\left(a-e_{\nu}\right)}{S^{2}(a)}\right)^{1 / 2} \frac{\vartheta_{1}(u) \vartheta_{4}\left(a_{\mu} \lambda-a_{\nu} \lambda-2 \lambda+u\right)}{\vartheta_{1}(3 \lambda) \vartheta_{4}\left(a_{\mu} \lambda-a_{\nu} \lambda+\lambda\right)} \\
& W\left(\begin{array}{cc}
a & a+e_{\mu} \\
a+e_{\mu} & a
\end{array}\right)=\frac{S\left(a+e_{\mu}\right)}{S(a)} \frac{\vartheta_{1}(u) \vartheta_{1}\left(2 a_{\mu} \lambda-2 \lambda+u\right)}{\vartheta_{1}(3 \lambda) \vartheta_{1}\left(2 a_{\mu} \lambda+\lambda\right)} \\
& +\frac{\vartheta_{1}(3 \lambda-u) \vartheta_{1}\left(2 a_{\mu} \lambda+\lambda+u\right)}{\vartheta_{1}(3 \lambda) \vartheta_{1}\left(2 a_{\mu} \lambda+\lambda\right)} \\
& W\left(\begin{array}{cc}
a & a+e_{\nu} \\
a+e_{\mu} & a+e_{\mu}+e_{\nu}
\end{array}\right) \\
& =\epsilon_{1}\left(\frac{\vartheta_{4}\left(a_{\mu} \lambda-a_{\nu} \lambda-\lambda\right) \vartheta_{4}\left(a_{\mu} \lambda-a_{\nu} \lambda+\lambda\right)}{\vartheta_{4}^{2}\left(a_{\mu} \lambda-a_{\nu} \lambda\right)}\right)^{1 / 2} \frac{\vartheta_{1}(u) \vartheta_{1}(3 \lambda-u)}{\vartheta_{1}(\lambda) \vartheta_{1}(3 \lambda)} \\
& S(a)=(-)^{a_{1}+a_{2}} \vartheta_{1}\left(2 a_{1} \lambda\right) \vartheta_{1}\left(2 a_{2} \lambda\right) \vartheta_{4}\left(a_{1} \lambda-a_{2} \lambda\right) \vartheta_{4}\left(a_{1} \lambda+a_{2} \lambda\right) \text {, }
\end{aligned}
$$

where $\nu \neq \pm \mu$ and $\lambda=\frac{\pi}{2} \frac{L}{L+1}$. Like the ABF model there are four different physical regimes:

$$
\begin{aligned}
& \left.\begin{array}{c}
0<p<1 \\
-1<p<0
\end{array}\right\} \quad 0<u<3 \lambda-\pi \quad \epsilon_{1}=-\epsilon_{2}=1 \\
& \left.\begin{array}{c}
0<p<1 \\
-1<p<0
\end{array}\right\} \quad 3 \lambda-2 \pi<u<0 \quad \epsilon_{1}=-\epsilon_{2}=-1
\end{aligned}
$$

Due to periodicity of the weights, the choice $\lambda=\frac{\pi}{2} \frac{L+2}{L+1}$, which also yields positive largest eigenvalues $\Lambda_{1}=\Lambda_{2}$, does not give any new regimes.

We note that, away from criticality, the above models break the $\mathrm{Z}_{2}$ symmetry of the underlying dynkin diagrams, when $L$ is odd. If we define $\tilde{a}_{i}=L+1-a_{i}$, we have

$$
W\left(\begin{array}{ll}
\left(d_{1}, d_{2}\right) & \left(c_{1}, c_{2}\right) \\
\left(a_{1}, a_{2}\right) & \left(b_{1}, b_{2}\right)
\end{array}\right) \neq W\left(\begin{array}{ll}
\left(\tilde{d}_{1}, d_{2}\right) & \left(\tilde{c}_{1}, c_{2}\right) \\
\left(\tilde{a}_{1}, a_{2}\right) & \left(\tilde{b}_{1}, b_{2}\right)
\end{array}\right) \quad L \text { odd }
$$

and similarly for the second component. We do however retain the symmetry

$$
W\left(\begin{array}{ll}
\left(d_{1}, d_{2}\right) & \left(c_{1}, c_{2}\right) \\
\left(a_{1}, a_{2}\right) & \left(b_{1}, b_{2}\right)
\end{array}\right)=W\left(\begin{array}{ll}
\left(\tilde{d}_{1}, \tilde{d}_{2}\right) & \left(\tilde{c}_{1}, \tilde{c}_{2}\right) \\
\left(\tilde{a}_{1}, \tilde{a}_{2}\right) & \left(\tilde{b}_{1}, \tilde{b}_{2}\right)
\end{array}\right)
$$

\section{Summary and discussion}

We have established a graphical equivalence between restricted solid-on-solid models and loop models. In particular, we have applied this equivalence to solvable loop models and, as a result, found new families of critical RSOS models. These new models can all be classified in terms of Dynkin diagrams of the simply-laced Lie 
algebras, the so-called A-D-E algebras. Furthermore we have indicated how to extend the equivalence to models that are of mixed loop-vertex type. Finally, an off-critical extension of the $\mathrm{C}_{2}^{(1)}$ RSOS model based on the Dynkin diagram pair $\left(\mathrm{A}_{L}, \mathrm{~A}_{L}\right)$ is given. This extension, which involves elliptic $\vartheta$-functions, breaks the $\mathrm{Z}_{2}$ symmetry off the underlying Dynkin diagrams.

Obvious generalisations of the ideas presented in this paper are:

(i) The extension to directed adjacency graphs, see e.g. [18].

(ii) The study of loop models that admit multiple occupation of edges. That is, each edge of the lattice can be occupied by more that one polygon segment, provided that all segments have different colour. Clearly the MCL-RSOS equivalence of section 3.2 still holds.

(iii) The extension to higher spin RSOS models, where we view the dense and dilute RSOS models as spin- $\frac{1}{2}$ and spin-1 models respectively.

(iv) The mapping of loop models onto RSOS-vertex or RSOS-SOS models. In section 3.2 we have shown how a loop model can be mapped onto a RSOS model by identifying the fugacity $n_{i}$ of a loop of colour $i$ with the largest eigenvalue $\Lambda_{i}$ of an adjacency matrix $A^{i}$. In section 5 we have shown how a loop model can be mapped onto a vertex model by setting $n_{i}=s_{i}^{4}+s_{i}^{-4}$, where the phase factor $s_{i}\left(s_{i}^{-1}\right)$ is associated with a directed loop making a turn to the left (right). Combining these two mapping, choosing $n_{i}=\Lambda_{i}\left(s_{i}^{4}+s_{i}^{-4}\right)$, we can map a loop model on a RSOS-vertex model or RSOS-SOS model. We note that this type of RSOS-SOS model is altogether different from the RSOS-SOS models defined in section 5 .

We hope to report a study of these generalisations in a future publications.

An intriguing open problem [15] is the relation between the critical $\mathrm{A}_{n}^{(1)}, \mathrm{B}_{n}^{(1)}$, $\mathrm{C}_{n}^{(1)}, \mathrm{D}_{n}^{(1)}, \mathrm{A}_{2 n}^{(2)}$ and $\mathrm{A}_{2 n-1}^{(2)}$ RSOS models found in [15] and 19 and their vertex couterparts given in 10$]$. For some of these models, notably the $\mathrm{A}_{1}^{(1)}, \mathrm{C}_{2}^{(1)}$ and $\mathrm{A}_{2}^{(2)}$ models, the MCL-RSOS equivalence, does provide a link between the RSOS and vertex representations. It remains unclear however, how to extend the methods of this paper to establish the RSOS-vertex correspondence in general.

\section{Acknowledgements}

We thank Paul A. Pearce for kind interest in our work. This work has been supported by the Stichting voor Fundamenteel Onderzoek der Materie (FOM).

\section{A Yang-Baxter equation for the MCL and RSOS model}

The equivalence between the MCL and RSOS model holds irrespective of the solvability of the models. In this appendix we show however that a sufficient condition 
for the YBE equation of the RSOS model to hold, is that the corresponding MCL model satisfies the YBE.

\section{A.1 Yang-Baxter equation for loop models}

Although loop models are intrinsically non-local, one can nevertheless formulate a local condition or YBE for two transfer matrices to commute [8]. (For the definition of the transfer matrix for loop models, see e.g. [17].) In order to define this equation we need some preliminaries.

Consider an object $\mathcal{O}$ with $p$ external edges, labelled $1, \ldots, p$, as shown in figure 6 . Edges of $\mathcal{O}$ can either be empty or occupied by a coloured polygon segment. Each edge that is occupied is, via the interior of $\mathcal{O}$, connected to a one other edge that is occupied by a line segement of equal colour. The index $\alpha_{k}$ contains the following information: (i) whether edge $k$ is occupied by a polygon segment of given colour, and (ii) if so, to which other edge it is connected. The information contained in all $\alpha_{1}, \ldots, \alpha_{p}$ is called the connectivity of $\mathcal{O}=\mathcal{O}\left(\alpha_{1}, \ldots, \alpha_{p}\right)$ and denoted by $C_{\mathcal{O}}$. The object $\mathcal{O}$ has a weight $W(\mathcal{O})$.

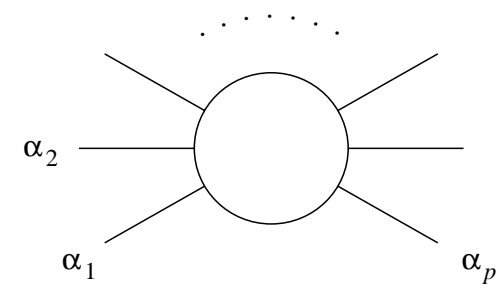

Figure 6: An object with $p$ external edges.

By the contraction of $\mathcal{A}$ and $\mathcal{B}$ to the composite object $\mathcal{D}$

$$
\begin{aligned}
\sum_{C_{\mathcal{A}}, C_{\mathcal{B}}} \mathcal{A}\left(\alpha_{1}, \ldots, \alpha_{k}, \mu_{1}, \ldots, \mu_{l}\right) \mathcal{B}\left(\beta_{1}, \ldots, \beta_{m}, \mu_{1}, \ldots, \mu_{l}\right) & \\
& =\mathcal{D}\left(\alpha_{1}, \ldots, \alpha_{k}, \beta_{1}, \ldots, \beta_{m}\right),
\end{aligned}
$$

we glue together the edges of $\mathcal{A}$ and $\mathcal{B}$ that carry the same index and sum over all connectivities of $\mathcal{A}$ and $\mathcal{B}$ consistent with $C_{\mathcal{D}}$. Here it is to be understood that $\mu_{i}$ in the argument of $\mathcal{A}$ and of $\mathcal{B}$ still signify two different things. We use the repeated occurence of the labels only to indicate that the edge of $\mathcal{A}$ that carries the index $\mu_{i}$ is glued to the edge of $\mathcal{B}$ that carries that same index. Furthermore it implies that edges which are glued together must be occupied by a polygon segment of the same colour. Finally it means that if the edge of $\mathcal{A}(\mathcal{B})$ carrying the index $\mu_{i}$ is occupied and connected to, say, the edge of $\mathcal{A}(\mathcal{B})$ carrying the index $\alpha_{j}\left(\beta_{k}\right)$ then then the edges of $\mathcal{D}$ carrying the indices $\alpha_{j}$ and $\beta_{k}$ are connected.

The weight $W(\mathcal{D})$ is defined as

$$
W(\mathcal{D}) \sum_{C_{\mathcal{A}}, C_{\mathcal{B}}} W(\mathcal{A}) W(\mathcal{B}) n_{1}^{p_{1}} \ldots n_{C}^{p_{C}}
$$

where $p_{i}$ is the number of polygons of colour $i$ that are closed by glueing together $\mathcal{A}$ and $\mathcal{B}$. 


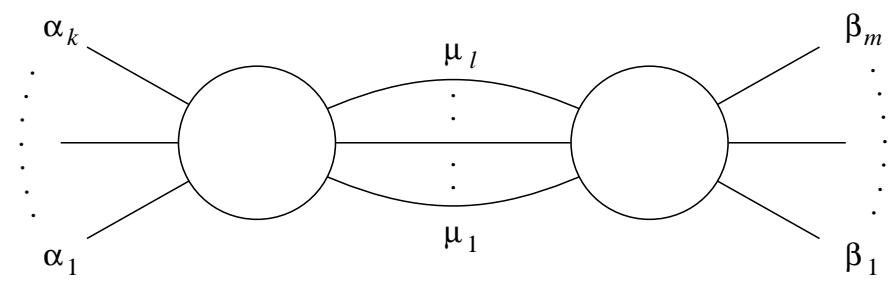

Figure 7: Graphical representation of the contraction of $\mathcal{A}$ and $\mathcal{B}$.

An elementary vertex of the MCL model has four external edges. Because the Boltzmann weight $W$ of a vertex $\mathcal{V}$ is completely determined by its connectivity, we can write

$$
W=W(\mathcal{V}(\alpha, \beta, \gamma, \delta)) \equiv W\left(\begin{array}{lll} 
& \delta & \\
\alpha & & \gamma \\
& \beta
\end{array}\right)
$$

With the above definitions, the YBE equation for the MCL model may be written as:

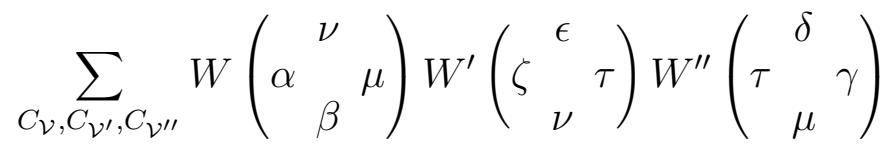

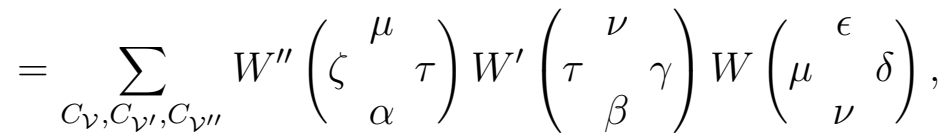

and must be satisfied for all possible conectivities $C_{\mathrm{YBE}(\alpha, \ldots, \zeta)}$. In other words, not only do we fix the occupation of the external edges, but also to which other external edge an occupied edge is connected. If one of the terms in the above equation has an internal loop of colour $i$ this yields a factor $n_{i}$.

\section{A.2 Yang-Baxter equation for the RSOS model}

To show that if the YBE for the MCL model is satisfied, it holds as well for the RSOS model, we begin with the YBE for the RSOS model [1]

$$
\begin{aligned}
\sum_{g} W\left(\begin{array}{ll}
f & g \\
a & b
\end{array}\right) W^{\prime} & \left(\begin{array}{ll}
e & d \\
f & g
\end{array}\right) W^{\prime \prime}\left(\begin{array}{ll}
d & c \\
g & b
\end{array}\right) \\
& =\sum_{g} W^{\prime \prime}\left(\begin{array}{ll}
e & g \\
f & a
\end{array}\right) W^{\prime}\left(\begin{array}{ll}
g & c \\
a & b
\end{array}\right) W\left(\begin{array}{ll}
e & d \\
g & c
\end{array}\right) .
\end{aligned}
$$

This equation must hold for all values of the external height vectors, with $a_{i}, \ldots, g_{i} \in$ $\left\{1, \ldots, L_{i}\right\}$. We substitute the definition of the weights $W, W^{\prime}$ and $W^{\prime \prime}$, where $W^{\prime}$ and $W^{\prime \prime}$ are given by (3.3) with $\rho_{k}$ replaced by $\rho_{k}^{\prime}$ and $\rho_{k}^{\prime \prime}$, and expand both sides of the YBE into $V^{3}$ terms. We then use the factorisation (3.2) and perform the trivial summation over the $\delta$-functions. As a result, most terms in the expansion 
no longer contain the variable $g_{i}$. Only terms for which the internal site differs in height from all its three neighbouring sites, which in that case all have equal height, yield a $g_{i}$ dependent factor of the form $S_{g_{i}} / S_{a_{i}}$. Here $g_{i}$ and $a_{i}$ are the $i$-th height components of the center site and its neighbouring sites respectively, and $a_{i} \sim g_{i}$, $a_{j}=g_{j} j \neq i$. Performing the sum over $g_{i}$ yields a factor $\Lambda_{i}$, see equation (3.10). We now group together all terms that have the same dependence on the vectors $S^{i}$. If we demand that the resulting equation holds for any arbitrary set of graphs $\left\{\mathcal{G}_{1}, \ldots, \mathcal{G}_{c}\right\}$, a sufficient and presumably necessary condition is that each group of terms vanishes independently. If we draw domain walls (of the appropriate colour) between regions of different height, terms within the same group all have the same connectivity. Furthermore, a term with an internal loop of colour $i$ contributes an extra factor $\Lambda_{i}$. As a result we find, upon setting $\Lambda_{i}=n_{i}$, that each group yields precisely one of the equations of the YBE for the MCL model.

\section{References}

[1] Baxter R J 1982 Exactly Solved Models in Statistical Mechanics (Academic: London)

[2] Pasquier V 1987 Nucl. Phys. B 285 [FS19] 162; 1987 J. Phys. A: Math. Gen. 20, L1229, 5707

[3] Owczarek A L and Baxter R J 1987 J Stat Phys 491093

[4] Warnaar S O, Nienhuis B and Seaton K A Phys. Rev. Lett. 69710

[5] Roche Ph 1992 Phys. Lett. B 28549

[6] Nienhuis B 1990 Int. J. Mod. Phys. B 4929

[7] Izergin A G and Korepin V E 1981 Comm. Math. Phys. 79303

[8] Nienhuis B, Warnaar S O and Blöte H W J 1993 J. Phys. A: Math. Gen. 26 ?

[9] Baxter R J, Kelland S B and Wu F Y 1976 J. Phys. A: Math. Gen. 9397

[10] Bazhanov V V 1985 Phys. Lett. 159B 321;

Jimbo M 1986 Commun. Math. Phys. 102537

[11] Cherednik I V 1980 Theor. Math. Phys. 43 356;

Chudnovsky D V and Chudnovsky G V 1980 Phys. Lett. 79A 36;

Kulish P P and Sklyanin E K 1980 Zapiski Nauch. Semin. LOMI 95 129;

Perk J H H and Schultz C L 1981 Phys. Lett. 84A 407

[12] van Beijeren H 1977 Phys. Rev. Lett. 38993

[13] Cvetkovic D M, Doob M and Sachs H 1980 Spectra of Graphs (Academic: London) 
[14] Andrews G E, Baxter R J and Forrester P J 1984 J. Stat. Phys. 35193

[15] Jimbo M, Miwa T and Okado M 1988 Commun. Math. Phys. 116507

[16] Gradshteyn I S and Ryzhik I M 1965 Tables of Integrals, Series and Products (Academic: New York)

[17] Blöte H W J and Nienhuis B 1989 J. Phys. A: Math. Gen. 221415

[18] Di Francesco P and Zuber J-B 1990 Nucl. Phys. B 338602

[19] Kuniba A, 1991 Nucl. Phys. B 355801 


\section{List of Figures}

1 (a) The 9 vertices of the $C=1$ MCL model and (b) the 23 vertices for the $C=2$ case. . . . . . . . . . . ...... 2

$2 \quad$ A polygon configuration. Only loops of different colour may intersect 6

$3 \quad$ The 20 vertices of the $\mathrm{A}_{3}^{2}$ loop-vertex model. . . . . . . . . . . . 8

$4 \quad$ Dynkin diagrams of the simply-laced Lie algebras. . . . . . . . . . . . . 10

$5 \quad$ Non-simple connected graphs with largest eigenvalue $\leq 2$. . . . . . . . 10

6 An object with $p$ external edges. . . . . . . . . . . . . . . 14

$7 \quad$ Graphical representation of the contraction of $\mathcal{A}$ and $\mathcal{B}$. . . . . . . . 15 